\title{
Oriented Morphometry of Folds on Surfaces
}

\author{
Maxime Boucher ${ }^{1, \star}$, Alan Evans ${ }^{2}$, and Kaleem Siddiqi ${ }^{1}$ \\ 1 School of Computer Science, McGill University, Canada \\ boucher@bic.mni.mcgill.ca \\ 2 McConnell Brain Imaging Center, Montreal Neurological Institute, McGill \\ University, Canada
}

\begin{abstract}
The exterior surface of the brain is characterized by a juxtaposition of crests and troughs that together form a folding pattern. The majority of the deformations that occur in the normal course of adult human development result in folds changing their length or width. Current statistical shape analysis methods cannot easily discriminate between these two cases. Using discrete exterior calculus and Tikhonov regularization, we develop a method to estimate a dense orientation field in the tangent space of a surface described by a triangulated mesh, in the direction of its folds. We then use this orientation field to distinguish between shape differences in the direction parallel to folds and those in the direction across them. We test the method quantitatively on synthetic data and qualitatively on a database consisting of segmented cortical surfaces of 92 healthy subjects and 97 subjects with Alzheimer's disease. The method estimates the correct fold directions and also indicates that the healthy and diseased subjects are distinguished by shape differences that are in the direction perpendicular to the underlying hippocampi, a finding which is consistent with the neuroscientific literature. These results demonstrate the importance of direction specific computational methods for shape analysis.
\end{abstract}

\section{Introduction}

The exterior surface of the human brain has a characteristic shape formed by a collection of folds. A fold can be described in loose terms as an oriented structure where the surface curvature has a greater magnitude in the direction perpendicular to its orientation than in the direction parallel to it. The study of the shape of folds on surfaces is particularly relevant in medical imaging. For example, it has been hypothesized by [1] that folds on the exterior surface of the brain are the result of the underlying mechanical tensions that force the surface to sink at certain locations. Thus, numerous mechanical models have been developed to analyze the shape of folding patterns and to find correlations among particular folds as a function of disease or other factors, such as age [2 [5].

\footnotetext{
^ Corresponding author.
} 
If one wishes to mathematically characterize the variability of surface shape in a population, a statistical shape model must be used and two ingredients are necessary. The first is a notion of the average shape and the second is an appropriate metric to provide a distance measure between two exemplars. In the case of brain imaging, the cortical surface is often represented as a single surface and continuous deformation models are built using diffeomorphic maps to match the fold on each exemplar surface with those on a population average. Several metrics has been proposed to describe how two surfaces are locally dissimilar (see e.g. 3, 6, 7] ). These metrics however lack specificity when it comes to differentiating between a deformation that occurs in the direction parallel to a fold and one in the direction perpendicular to it. Certain shape differences, such as a relative depth difference between two folds, can only occur when the spacing between two consecutive crests is altered. Thus, in the context of medical image analysis, the interpretation of the results can strongly depend on the orientation of the shape deformation.

To address the above problem, we develop a method to estimate a dense orientation field on a surface, such that it is optimally oriented along the direction of its folds 1 . A fold on a surface is comprised by locations where the principal curvature is high in the direction perpendicular to it, and is low in the direction along it. We thus use this description of a fold to estimate an orientation field using Tikhonov regularization. The approach we develop is motivated by the literature on smoothing direction fields in images, such as the techniques in 8 10]. These methods cannot be directly applied to our problem. For example, in the method in [8] the underlying vector field is embedded in Euclidean space whereas we need to embed the orientation field into the tangent space of the surface. For this purpose, we use the discrete exterior calculus framework of [11] to develop a simple computational method for a discrete triangulated mesh. The triangles provide an estimate of the tangent space and the orientation field is embedded within each triangle. The mesh surface is then unfolded locally to solve the variational problem. Once the orientation field is found, we provide a statistical measure of shape difference to determine if two folds differ in length or in width.

We validate our approach quantitatively on synthetic surfaces, where the underlying ground truth fold orientation is known, and qualitatively on a database comprised of a combination of cortical surfaces of healthy subjects and those of patients with Alzheimer's disease. We find that on a surface with known groundtruth, the estimated orientation fields follow the expected orientation. We then provide a metric for oriented shape differences and test this metric on the population affected by Alzheimer's disease. We find that the hippocampi are atrophied $(p<0.001)$ in the direction perpendicular to an associated fold, which is consistent with the neuroscientific literature. Our results thus imply that increased specificity is achieved by orienting the analysis of shape difference.

${ }^{1}$ In this paper, a direction field is a vector field where all vectors are constrained to have unit length. An orientation field is a direction field where opposing vectors are considered equivalent. 


\section{Finding the Orientation of Folds}

We begin by developing an energy functional which when minimized results in a smooth orientation field on a surface, which follows the direction of a folding pattern. To get an intuitive sense of our goals, consider Fig. 1 which illustrates a portion of a cortical surface. A crest (or ridge) is shown in red, with a nearby parallel trough (or valley) in yellow. A fold is characterized by a juxtaposition of crests and troughs with the same orientation. In the case of the human brain this is an approximation that is correct for the most part, except at pattern singularities. The dense orientation field estimated by our method corresponds to the local orientation of crests and troughs. In Section 2.2 we describe how our solution can be discretized and solved numerically on a triangulated mesh. Then, in Section 2.3, we present a statistical shape model which uses the estimated orientation field to differentiate between shape differences which occur in the direction of a fold and those which occur across it.

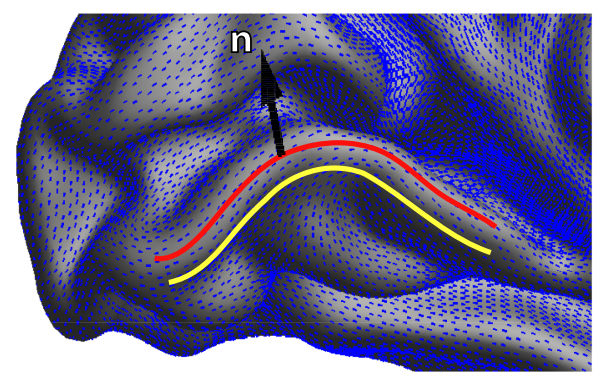

Fig. 1. A dense orientation field that follows crests and troughs

\subsection{A Minimum Principal Curvature Orientation Field}

We first develop a mathematical model of a fold on a surface. Consider a Riemannian manifold $(\mathcal{S}, g)$, where $g$ is a Riemannian metric. Let the unit normal vector to the surface be $\mathbf{n}$. Let $\mathbf{v}$ be a vector field tangent to the surface $\mathcal{S}$ and let $\nabla$ be the torsion-free affine connection on $\mathcal{S}$. Using this notation, the associated covariant derivative along $\mathbf{v}$ is $\nabla_{\mathbf{v}}$ and the shape operator $S(\mathbf{v})$ is given by $\nabla_{\mathbf{v}} \mathbf{n}$. Covariant derivation being a linear operation, with a slight abuse in notation the action of the shape operator $S$ on the vector field can be expressed as

$$
S(\mathbf{v})=S \mathbf{v} .
$$

Because the affine connection $\nabla$ is torsion free, the second fundamental form is symmetric, i.e., $S=S^{t}$.

We first define an orientation field on a surface and provide a differentiable metric on orientation fields, which will later be used to set up an appropriate variation problem. Let a unit-norm vector field within the tangent space of the surface $\mathcal{S}$ be defined such that the norm of any vector is equal to 1 :

$$
\mathbf{v}: \mathcal{S} \mapsto \mathcal{T}_{\mathbf{p}} \mathcal{S},\|\mathbf{v}(\mathbf{p})\|=1
$$


We define an orientation field as a unit-norm vector field which is invariant under rotations of $\pi$ radians. For example, the structure tensor, defined as

$$
M(\mathbf{v})=\mathbf{v} \otimes \mathbf{v},
$$

is invariant under rotations of $\pi$ radians $(M(\mathbf{v})=M(-\mathbf{v}))$. It is often more convenient to parametrize the space of possible orientations using angles $\theta$. A unit length vector $\mathbf{v}$ is then expressed as $\mathbf{v}_{\theta}=(\cos (\theta), \sin (\theta))$. The $\theta$ parameterization allows us to define a natural Riemannian metric between two orientations $\mathbf{v}_{\theta_{1}}$ and $\mathbf{v}_{\theta_{2}}$ as

$$
d_{\text {Riemann }}\left(\mathbf{v}_{\theta_{1}}, \mathbf{v}_{\theta_{2}}\right)^{2}=\min _{n}\left(\theta_{1}-\theta_{2}+n \pi\right)^{2}, n \in \mathbb{Z} .
$$

However, this metric lacks differentiability at the point $\theta_{1}-\theta_{2}+n \pi=\pi / 2$. In practice, the lack of differentiability makes it difficult to find a global minimum of a variational problem using a gradient descent approach. For this reason, we follow the approach described in [8, 9] which is to approximate the Riemannian metric by a differentiable metric which is locally equivalent to Equation 4 if the orientation field is continuous. Let the structure tensor be given as

$$
M\left(\mathbf{v}_{\theta}\right)=\left(\begin{array}{cc}
\cos ^{2}(\theta) & \sin (\theta) \cos (\theta) \\
\sin (\theta) \cos (\theta) & \sin ^{2}(\theta)
\end{array}\right) .
$$

Let $M_{\theta}=M\left(\mathbf{v}_{\theta}\right)$, and let a basis vector of the tangent space of $M_{\theta}$ be given as

$$
T_{\theta}=\frac{\partial M_{\theta}}{\partial \theta}=\left(\begin{array}{cc}
-\sin (2 \theta) & \cos (2 \theta) \\
\cos (2 \theta) & \sin (2 \theta)
\end{array}\right) .
$$

Let the contraction : be defined between two matrices $A$ and $B$ as $A: B=$ trace $\left(A^{t} B\right)$ and the associated Frobenius norm defined as $\|A\|_{F}^{2}=A: A$. We define the Euclidean orthonormal projection of $M_{\theta_{2}}$ onto the tangent space of $M_{\theta_{1}}$ as

$$
\Pi_{M_{\theta_{1}}}\left(M_{\theta_{2}}\right)=M_{\theta_{2}}-T_{\theta_{1}} \frac{T_{\theta_{1}}: M_{\theta_{2}}}{\left\|T_{\theta_{1}}\right\|_{F}^{2}} .
$$

We then define a differentiable metric between $M_{1}$ and $M_{2}$ as

$$
d\left(M_{\theta_{1}}, M_{\theta_{2}}\right)=\frac{1}{2}\left\|\Pi_{M_{\theta_{1}}}\left(M_{\theta_{2}}\right)\right\|_{F}
$$

The following theorem shows $d\left(M_{\theta_{1}}, M_{\theta_{2}}\right)$ and $d_{\text {Riemann }}\left(\mathbf{v}_{\theta_{1}}, \mathbf{v}_{\theta_{2}}\right)$ are locally equivalent if the orientation field is continuous.

\section{Theorem 21}

$$
\lim _{\theta_{2} \rightarrow \theta_{1}} \frac{d\left(M_{\theta_{1}}, M_{\theta_{2}}\right)}{d_{\text {Riemann }}\left(\mathbf{v}_{\theta_{1}}, \mathbf{v}_{\theta_{2}}\right)}=1
$$

Proof. Choose $\theta_{2}^{\prime}=\theta_{2}+n \pi, n \in \mathbb{Z}$ such that $\left(\theta_{1}-\theta_{2}^{\prime}+n \pi\right)^{2}$ is minimum for $n=0$ and perform a Taylor series expansion of $d\left(M_{\theta_{1}}, M_{\theta_{2}^{\prime}}\right)$ around $M_{\theta_{1}}$ to obtain that

$$
d\left(M_{\theta_{1}}, M_{\theta_{2}}\right)=d_{\text {Riemann }}\left(\mathbf{v}_{\theta_{1}}, \mathbf{v}_{\theta_{2}}\right)\left(1+\mathcal{O}\left(d_{\text {Riemann }}\left(\mathbf{v}_{\theta_{1}}, \mathbf{v}_{\theta_{2}}\right)^{2}\right)\right) .
$$

Thus, in the limit, the two metrics are equivalent. 
We now use this differentiable structure to set up a variational problem whose solution will provide a direction field oriented along the crest lines and trough lines of a surface. A crest on a surface is given by a curve $\gamma \subset \mathcal{S}$ such that the curvature of $\mathcal{S}$ in the direction perpendicular to $\gamma$ is positive, and reaches a maximum in magnitude. A trough has the same property, except that the sign of the curvature in the direction perpendicular to $\gamma$ is now negative. Hence, to estimate the presence of a fold in a given orientation we define the following surface curvature energy function

$$
E_{\text {curvature }}\left(\mathbf{v}_{\theta}\right)=\int_{\mathcal{S}}\left\|S \mathbf{v}_{\theta}\right\|^{2} d \mathcal{S} .
$$

If $\lambda_{1}^{2}<\lambda_{2}^{2}$ are the eigenvalues of $S^{t} S$ with associated eigenvectors $\mathbf{v}_{1}, \mathbf{v}_{2}$, then the minimum value of $E_{\text {curvature }}$ is achieved for $\mathbf{v}=\mathbf{v}_{1}$.

A fold is characterized by a juxtaposition of crests and troughs with the same orientation (see Fig. 11). To capture this idea, we define a second energy functional which measures the local smoothness of the orientation field

$$
E_{\text {smoothness }}\left(M_{\theta}\right)=\int_{\mathcal{S}}\left\|\nabla M_{\theta}\right\|^{2} d \mathcal{S} .
$$

We will deem a crest $\gamma_{1}$ and a trough $\gamma_{2}$ to have roughly the same orientation if there is an orientation field $\mathbf{v}$ on $\mathcal{S}$ such that $\mathbf{v}$ is tangent to both $\gamma_{1}$ and $\gamma_{2}$ and such that the energy functional $E_{\text {smoothness }}\left(M_{\theta}\right)$ is small. Our goal is not to explicitly locate the crests and troughs but rather to directly find an orientation field that minimizes both Equations 9 and 10. This leads to the following estimate for a fold orientation, based on a regularization functional:

$$
M_{\theta}=\operatorname{argmin}_{M_{\theta}^{*}}\left(\alpha E_{\text {smoothness }}\left(M_{\theta}^{*}\right)+E_{\text {curvature }}\left(M_{\theta}^{*}\right)\right) .
$$

Here $E_{\text {smoothness }}$ is the smoothness term, $E_{\text {curvature }}$ is the data attachment term and $\alpha$ is a constant to balance to the two.

We can then find the first variation of Equation 11 to find a minimum via the gradient descent equation

$$
\frac{\partial M_{\theta}}{\partial t}=\Pi_{M_{\theta}}\left(\alpha \Delta_{\mathcal{S}} M_{\theta}-S^{t} S\right)
$$

\subsection{Discrete Heat Diffusion of the Orientation Field on a Triangulated Mesh}

To solve Equation 11 on a discrete triangulated mesh, we need to be able to smooth an orientation field on a surface. The initial orientation field is given by the eigendirection with lowest absolute eigenvalue of the shape operator. We thus first describe an expression for the shape operator $S$ and then provide a numerical scheme to diffuse the orientation field on each triangle. We assume that the orientation field is constant over each triangle and use finite volume methods. Since the normal vector of a triangle is constant on it, the tangent space of a 
triangle is uniquely defined. More importantly, we can use parallel transport to transport the orientation field between any two neighboring triangles in a unique fashion.

The shape operator simply describes the rate of change of the unit normal between two neighboring triangles, as explained in [12]. On an orientable triangulated mesh, the unit normal vector is uniquely defined for every triangle, by choosing one of the two possible directions. Let the set of all triangles be denoted $\left\{\triangle_{i}, i=1, \ldots, N\right\}$ and let $\mathbf{p}_{i, j}, j=1,2,3$, be the three vertices of triangle $\triangle_{i}$. We denote the oriented edges of the triangle as $\mathbf{l}_{i, 1}=\mathbf{p}_{i, 2}-\mathbf{p}_{i, 1}, \mathbf{l}_{i, 2}=$ $\mathbf{p}_{i, 3}-\mathbf{p}_{i, 2}, \mathbf{l}_{i, 3}=\mathbf{p}_{i, 1}-\mathbf{p}_{i, 3}$ and by $\mathbf{e}_{i, j}$ the unit normal vector that points in the same direction as $\mathbf{1}_{i, j}$. We denote by $\mathbf{n}_{i}$ the unit normal of the triangle and by $\mathbf{n}_{i, j}, j=1,2,3$ the unit normals of the neighboring triangles (those with which an edge is shared). Finally, let $A_{i}$ be the area of the triangle and $\mathbf{e}_{i, j}^{\perp}$ the unit outward pointing vector that is perpendicular to both $\mathbf{e}_{i, j}$ and $\mathbf{n}_{i}$. The shape operator $S_{i}$ then measures the difference in the orientation of the normal vectors $\mathbf{n}_{i}$ with the one of its neighboring triangles $\mathbf{n}_{i, j}$ weighted by length of the adjacent edges [12]:

$$
S_{i}=\frac{1}{A_{i}} \sum_{j \in\{1,2,3\}}\left\|\mathbf{l}_{i, j}\right\| M\left(\mathbf{e}_{i, j}^{\perp}\right)<\mathbf{e}_{i, j}^{\perp}, \mathbf{n}_{i, j}-\mathbf{n}_{i}>.
$$

We now need to diffuse the orientation field on a discrete triangulated surface so that it is restricted to the tangent space of a surface. The solution we propose to this problem is to locally unfold the surface around a triangle to place the neighboring triangles within the same Euclidean plane. Once the solution is computed, it can be mapped back onto the original surface. We write the orientation vector $M_{\theta}$ as a general Euclidean tensor in $\mathbb{R}^{3} \times \mathbb{R}^{3}$. Suppose that $M_{\theta, i}$ is our current estimate at $\triangle_{i}$ and $M_{\theta, i, j}, j=1,2,3$ is the estimate at the neighboring triangles. To locally unfold the surface, we need to rotate the estimate at the neighboring triangles $M_{\theta, i, j}$ such that they lie in the same plane as $M_{\theta, i}$. To do this, we need to pick a rotation matrix such that $\mathbf{n}_{i, j}$ is mapped onto $\mathbf{n}_{i}$. Also, we already know that an edge $\mathbf{e}_{i, j}$ that separates two triangles is already in the tangent space of both triangles and hence should remain fixed. These two constraints uniquely define the orientation matrix that maps the tangent space of neighboring triangles:

$$
R_{i, j}=\mathbf{e}_{i, j} \otimes \mathbf{e}_{i, j}+\mathbf{n}_{i} \otimes \mathbf{n}_{i, j}+\left(\mathbf{e}_{i, j} \times \mathbf{n}_{i}\right) \otimes\left(\mathbf{e}_{i, j} \times \mathbf{n}_{i, j}\right) .
$$

Obviously, since the orientation field is always perpendicular to the normal vector to the triangle, it is not necessary to rotate the entire space, but only the plane that contains the triangle. On $\triangle_{i}$, we use local coordinates $\mathbf{u}_{i, k}, k=1,2$ such that $\mathbf{u}_{i, 1} \times \mathbf{u}_{i, 2}=\mathbf{n}_{i}$, as a basis to describe the orientation field. We express the orientation field using the $\mathbf{u}_{i, j}$ coordinates for each triangle. Let $\mathbf{u}_{i, j, k}$ be the coordinate frame of the triangle $\triangle_{i, j}$. Then the rotation matrix between the tangent space of $\triangle_{i}$ and $\triangle_{i, j}$ is given as

$$
R_{i, j}=\left[\mathbf{u}_{i, 1}, \mathbf{u}_{i, 2}\right]\left(\mathbf{e}_{i, j} \otimes \mathbf{e}_{i, j}+\left(\mathbf{e}_{i, j} \times \mathbf{n}_{i}\right) \otimes\left(\mathbf{e}_{i, j} \times \mathbf{n}_{i, j}\right)\right)\left[\mathbf{u}_{i, j, 1}, \mathbf{u}_{i, j, 2}\right]^{t}
$$


To perform diffusion on a triangulated mesh, we also need a discrete LaplaceBeltrami operator. In the case of a scalar density field over a triangle, an expression for the discrete Laplace-Beltrami operator in terms of differences between the value of the density field at $\triangle_{i}$ and its neighboring triangles is given in [12]. Let $A_{i, j}$ be the area of triangle $\triangle_{i, j}$ and let $F$ be a scalar function on the mesh such that $F\left(\triangle_{i}\right)=F_{i}$. We denote by $\beta_{i, j}$ and $\gamma_{i, j}$ the opposite angles of triangles $\triangle_{i}$ and $\triangle_{i, j}$. Then, the discrete Laplace-Beltrami operator is

$$
\left(\Delta_{\mathcal{S}} F\right)_{i}=\frac{1}{A_{i}} \sum_{j=1}^{3} \frac{1}{\cot \left(\beta_{i, j}\right)+\cot \left(\gamma_{i, j}\right)}\left(F_{i}-F_{i, j}\right) .
$$

If we write $\mathbf{F}=\left(F_{1}, \ldots, F_{N}\right)^{t}$ as a vector, over a discrete triangular mesh $\Delta_{\mathcal{S}}$ can be written as a product of two matrices $\Delta_{\mathcal{S}}=A^{-1} L$, where $A$ is a diagonal matrix whose diagonal entries are given by $A_{i}$ and $L$ is a sparse positive semidefinite symmetric matrix whose entries are given by Equation 16

We can now express the Laplace-Beltrami operator for an orientation field using the rotation matrices given by Equation [15.

$$
\left(\Delta_{\mathcal{S}} M_{\theta}\right)_{i}=\frac{1}{A_{i}} \sum_{j=1}^{3} \frac{1}{\cot \left(\beta_{i, j}\right)+\cot \left(\gamma_{i, j}\right)}\left(M_{\theta, i}-R_{i, j} M_{\theta, i, j} R_{i, j}^{t}\right) .
$$

Now let $M_{\theta, i}$ be expressed as a vector of coordinates $a_{1} \mathbf{u}_{i, 1} \otimes \mathbf{u}_{i, 1}+b_{i}\left(\mathbf{u}_{i, 1} \otimes \mathbf{u}_{i, 2}+\right.$ $\left.\mathbf{u}_{i, 2} \otimes \mathbf{u}_{i, 1}\right)+c_{i} \mathbf{u}_{i, 2} \otimes \mathbf{u}_{i, 2}$. Then, if we write $\mathbf{M}_{\theta}=\left[a_{1}, b_{1}, c_{1}, \ldots, a_{N}, b_{N}, c_{N}\right]$, $\Delta_{\mathcal{S}}$ can be written as $\Delta_{\mathcal{S}}=A^{-1} L$ with $L$ a sparse symmetric positive semidefinite matrix with entries given by Equation [17. This provides us with an efficient numerical scheme to find a minimum of the variational problem given in Equation 11.

\subsection{An Oriented Shape Deformation Model}

Once a dense orientation field $\mathbf{v}$ in the direction of folds has been found it is possible to develop a shape deformation model that is specific to deformations that occur either along the orientation of a fold or in the direction parallel to it. Assume that we have a set of surfaces $\mathcal{S}_{l}, l=1, \ldots, n$ with a set of diffeomorphisms that map these surfaces onto a template average $\overline{\mathcal{S}}$ :

$$
\phi_{l}: \mathcal{S}_{l} \rightarrow \overline{\mathcal{S}}
$$

Examples of algorithms to find diffeomorphisms that match the shape of the surfaces based on the folding patterns can be found in [4, 13]. Suppose that $\bar{\triangle}_{i}$ is a triangle on $\overline{\mathcal{S}}$ and $\phi_{l}^{-1}$ is its mapping on $\mathcal{S}_{l}$. We note by $D \overline{\mathcal{S}}$ the differential in the triangle's coordinate, given as

$$
D_{i} \overline{\mathcal{S}}=\left[\overline{\mathbf{l}}_{i, 1}, \overline{\mathbf{l}}_{i, 2}\right] .
$$

The grad operator, given by the dual of the differential is

$$
\nabla_{i} \overline{\mathcal{S}}=\left(D_{i} \overline{\mathcal{S}}^{t} D_{i} \overline{\mathcal{S}}\right)^{-1} D_{i} \overline{\mathcal{S}}^{t}
$$


The dilation of the triangle $\bar{\triangle}_{i}$ by the diffeomorphism $\phi_{l}$ is then given by

$$
D_{i, l}=\left(D_{i} \mathcal{S}_{l} \nabla_{i} \overline{\mathcal{S}}\right)^{t}\left(D_{i} \mathcal{S}_{l} \nabla_{i} \overline{\mathcal{S}}\right)
$$

The directional dilatation is then given as

$$
D_{i, l, \mathbf{v}}=\mathbf{v}_{i, \text { euc }}^{t} D_{i, l} \mathbf{v}_{i, \text { euc }}
$$

where $\mathbf{v}_{i, \text { euc }}$ is the projection of the tangent vector onto the corresponding $3 \times 1$ vector in Euclidean space $\mathbb{R}^{3}$. From Equation 21, we see that $D_{i, l, \mathbf{v}}$ is always positive. This means that the dilation can be modeled using a log-normal distribution as

$$
\left.\log \left(D_{i, l, \mathbf{v}}\right) \sim \widehat{\log \left(D_{i, \mathbf{v}}\right.}\right)+\epsilon_{i, l}
$$

where $\widehat{\log \left(D_{i, \mathbf{v}}\right)}$ is the average dilation over all surfaces $\mathcal{S}_{l}$ and $\epsilon_{i, l}$ is a random variable which is normally distributed and has finite variance. The likelihood of such a model can be determined using a standard Student $t$-test while correcting for multiple comparisons, using the method described in [14].

\section{Results}

In this section we validate our method on synthetic data, for which the ground truth (desired) orientation field is known. We then present experimental results on a population of surfaces extracted from MRI scans of elderly humans.

\subsection{Quantitative Validation}

We created a circle relief on a flat background as our first test surface (see Fig. 2(a). On this surface, we expect that the detected fold orientation will be tangential to the radial direction. To generate the figure, we used a simple grayscale image of the desired circle relief and randomly placed points on the resulting height function. These points were then triangulated using the approach of [15]. A second "T" relief surface, shown in Fig. 2(b)] was also used. On this surface, we expect that the detected fold orientation will be tangential to the edges of the "T" . Equation 12 was solved on both of these surfaces. We stopped the simulation whenever the following ratio fell below a chosen threshold:

$$
\frac{\sup \left(\left\|\Pi_{M_{\theta}}\left(\alpha \Delta_{\mathcal{S}} M_{\theta}-S^{t} S\right)\right\|\right)}{\sup \left(\left\|\Pi_{M_{\theta}}\left(\alpha \Delta_{\mathcal{S}} M_{\theta}\right)\right\|+\left\|\Pi_{M_{\theta}}\left(S^{t} S\right)\right\|\right)}<\epsilon .
$$

For the circle relief, we initialized our algorithm using random orientations. We picked a constant of $\alpha=10$ for the smoothness term and an $\epsilon$ of $1 e-4$ in Equation 24. Visual results for the circle relief are shown in Fig. 2(c) The colormap shows the difference between the estimated orientation of the fold and the expected orientation. The recovered orientation field is accurate everywhere, with the exception of the center of the relief, where the notion of a fold orientation is not evident. On Fig. 2(a) we see there are two singularities in opposition to 


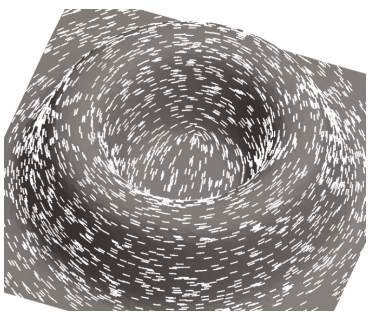

(a) Recovered Fold Orientation for the Circle Relief

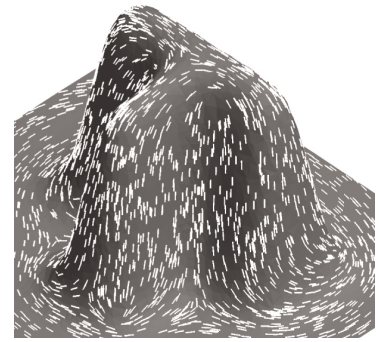

(b) Recovered Fold Orientation for the $\mathrm{T}$ Relief

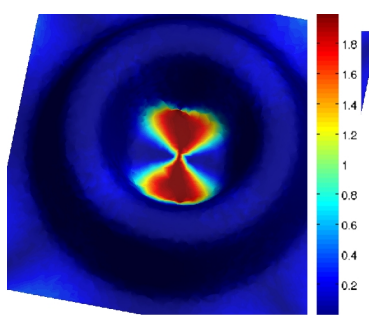

(c) Accuracy of Fold Orientation for the Circle Relief (in radians)

Fig. 2. Validation on synthetic surfaces

one another, which gives an arbitrary orientation. However, on cortical surfaces, most orientation singularities would arise from junctions of prominent crests and troughs. For the second synthetic surface, the results are shown in Fig. 2(b) The method recovers the expected orientations, as well as a singularity at the junction of the three segments of the "T".

\subsection{Results on Cortical Surfaces}

We used the OASIS database, which consists of 97 healthy subjects and 92 subjects (aged 60 and above) affected with mild and very-mild dementia [16]. We used the same surface extraction pipeline as in [13, 17, 18], which produces one mid-surface representation of the gray-matter cortical sheet, and obtained mappings $\phi_{l}$ for each surface onto a common template average. As with every surface analysis algorithm, mesh resolution can be a concern. Validations of the mesh generation and registration technique can be found in [13, 17, 18]. Once this mapping was found, we computed the average surface of the entire population and used this average surface to compute orientation fields.

To compute the fold orientation, we solved Equation 11 using both $\alpha=1$ and $\alpha=100$. A close up result for the left hemisphere is shown in Figure 3, while a result for the right hemisphere is shown in Figure 1. As can be visually assessed on Figure 3, the estimated orientations accurately follow the folds of the surface. For $\alpha=1$, the recovered orientation field is less smooth and closely follows every ridge on the surface. For $\alpha=100$, however, the orientation field is smoothed out and folds that are separated by a small ridge are connected. This allows us to obtain a global picture of the folding pattern of the surface, which is used for oriented shape analysis in the following section.

\subsection{Oriented Deformation Morphometry}

The direction of the fold on a surface allows us to test if its shape is influenced by an exterior factor, such as a particular disease. For example, in the neuroscience 


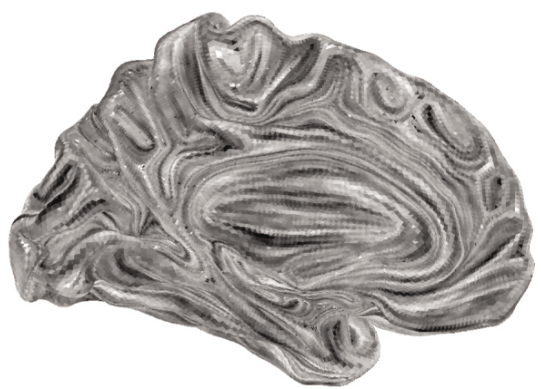

(a) Visualization of the orientation field (b) Visualization of the orientation field for $\alpha=1$

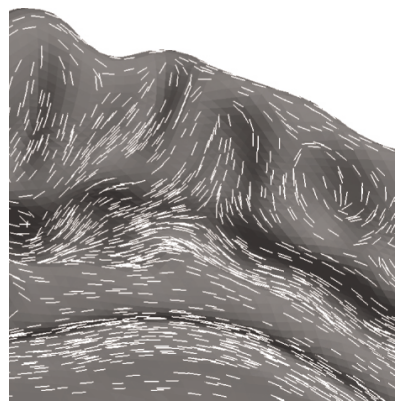

(c) Close up of the field, $\alpha=1$

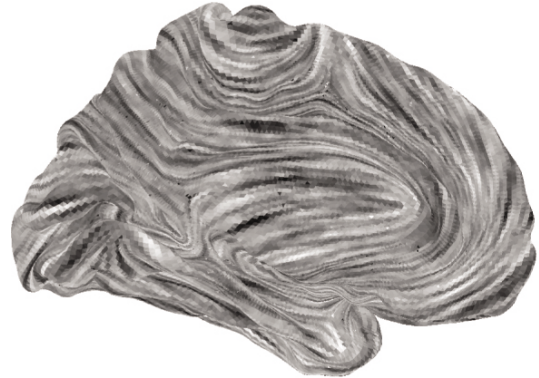
for $\alpha=100$

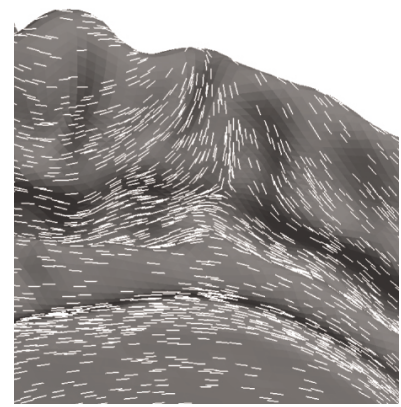

(d) Close up of the field, $\alpha=100$

Fig. 3. Estimated Fold Orientation in a saggital cut of the left hemisphere. The orientation field is smoother for $\alpha=100$. Visualization of the orientation field produced by integrating a random image along the orientation field (see [19]).

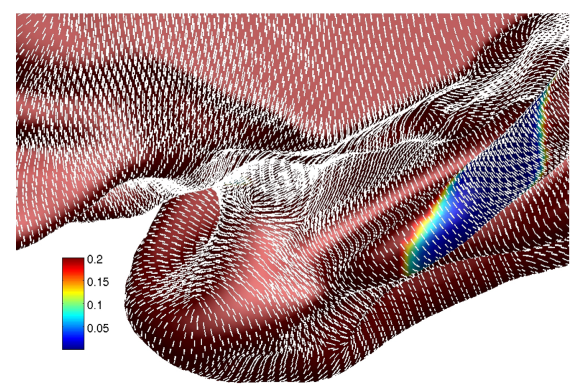

(a) Interior Right Temporal Lobe $(p<0.001)$

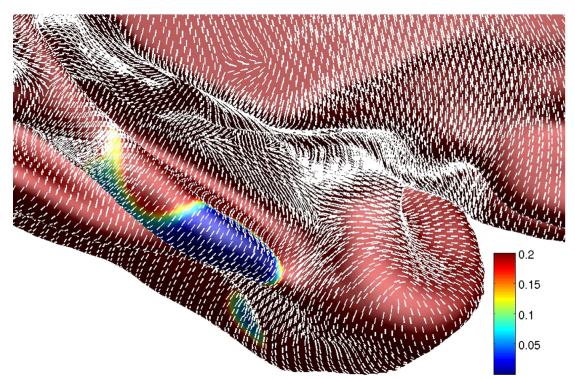

(b) Interior Left Temporal Lobe $\left(p<3 \times 10^{-5}\right)$

Fig. 4. Shape difference perpendicular to fold orientation due to Alzheimer's disease. The perpendicular orientation field is shown with its $p$ value as a colorbar. 
literature, it is well known that regions such as the hippocampus are among the first to be affected by Alzheimer's disease [2]. Our estimate of orientation on surfaces allows us to determine the nature of the deformation on the cortical surface. As explained in the introduction, a shorter fold corresponds to a deformation parallel to the fold orientation, whereas a shallower one corresponds to a deformation perpendicular to the fold. We used Equation 22 to produce two deformation maps, one that is parallel to the folds, and one that is perpendicular to them. We then performed a T-test using Equation 23 to compute probability $(p)$ values, after correcting for age, gender and multiple comparisons [14. We found that the main shape differences occur in the direction perpendicular to the folds, as illustrated in Figure 4. These results indicate that atrophy on the cortical surface may have preferential direction. We point out that it would not be possible to assess such changes using methods such as [3, 7], which lack direction specificity.

\section{Conclusion}

We have described a method to estimate a dense orientation field in the direction of folds on a surface. The method is based on a regularization functional and a numerical implementation that works directly on a triangulated mesh. The experimental results indicate that this method has great promise. In particular, for synthetic data the obtained orientation field is quantitatively accurate and for a database of cortical surfaces the method allows us to distinguish between direction specific shape changes.

\section{Acknowledgments}

This work was supported by funds from NSERC, FQRNT and CIHR.

\section{References}

1. Van Essen, D.: A tension-based theory of morphogenesis and compact wiring in the central nervous system. Nature 385, 313-318 (1997)

2. Buckner, R.: Memory and Executive Function in Aging and AD Multiple Factors that Cause Decline and Reserve Factors that Compensate. Neuron 44(1), 195-208 (2004)

3. Chung, M., Worsley, K., Robbins, S., Paus, T., Taylor, J., Giedd, J., Rapoport, J., Evans, A.: Deformation-based surface morphometry applied to gray matter deformation. NeuroImage 18(2), 198-213 (2003)

4. Fischl, B., Sereno, M., Dale, A.: Cortical surface-based analysis. II: Inflation, flattening, and a surface-based coordinate system. Neuroimage 9(2), 195-207 (1999)

5. Toga, A., Thompson, P.: Mapping brain asymmetry. Nature Reviews Neuroscience 4(1), 37-48 (2003)

6. Fillard, P., Arsigny, V., Ayache, N., Pennec, X.: A Riemannian Framework for the Processing of Tensor-Valued Images. In: Fogh Olsen, O., Florack, L.M.J., Kuijper, A. (eds.) DSSCV 2005. LNCS, vol. 3753, pp. 112-123. Springer, Heidelberg (2005) 
7. Lepore, N., Brun, C., Chou, Y., Chiang, M., Dutton, R., Hayashi, K., Luders, E., Lopez, O., Aizenstein, H., Toga, A., et al.: Generalized Tensor-Based Morphometry of HIV/AIDS Using Multivariate Statistics on Deformation Tensors. IEEE Transactions on Medical Imaging 27(1), 129-141 (2008)

8. Tang, B., Sapiro, G., Caselles, V.: Diffusion of General Data on Non-Flat Manifolds via Harmonic Maps Theory: The Direction Diffusion Case. International Journal of Computer Vision 36(2), 149-161 (2000)

9. Perona, P.: Orientation diffusions. IEEE Image Processing 7, 457-467 (1998)

10. Kimmel, R., Sochen, N.: Orientation Diffusion or How to Comb a Porcupine. Visual Com. and Image Representation 13(1-2), 238-248 (2002)

11. Hirani, A.: Discrete exterior calculus. PhD thesis, California Institute of Technology (2003)

12. Grinspun, E., Gingold, Y., Reisman, J., Zorin, D.: Computing discrete shape operators on general meshes. In: Computer Graphics Forum, vol. 25, pp. 547-556. Blackwell Synergy, Malden (2006)

13. Lyttelton, O., Boucher, M., Robbins, S., Evans, A.: An unbiased iterative group registration template for cortical surface analysis. Neuroimage 34(4), 1535-1544 (2007)

14. Worsley, K., Andermann, M., Koulis, T., MacDonald, D., Evans, A.: Detecting changes in nonisotropic images. Human Brain Mapping 8(2-3), 98-101 (1999)

15. Fisher, M., Springborn, B., Schröder, P., Bobenko, A.: An algorithm for the construction of intrinsic delaunay triangulations with applications to digital geometry processing. Computing 81(2), 199-213 (2007)

16. Marcus, D., Wang, T., Parker, J., Csernansky, J., Morris, J., Buckner, R.: Open Access Series of Imaging Studies (OASIS): Cross-sectional MRI Data in Young, Middle Aged, Nondemented, and Demented Older Adults. Journal of Cognitive Neuroscience 19(9), 1498-1507 (2007)

17. Boucher, M., Whitesides, S., Evans, A.: Depth potential function for folding pattern representation, registration and analysis. Medical Image Analysis (2008)

18. Kim, J., Singh, V., Lee, J., Lerch, J., Ad-Dabbagh, Y., MacDonald, D., Lee, J., Kim, S., Evans, A.: Automated 3-D extraction and evaluation of the inner and outer cortical surfaces using a Laplacian map and partial volume effect classification. Neuroimage 27, 210-221 (2005)

19. Cabral, B., Leedom, L.: Imaging vector fields using line integral convolution. In: Proceedings of the 20th annual conference on Computer graphics and interactive techniques, pp. 263-270. ACM, New York (1993) 